Check for updates

Cite this: Chem. Commun., 2020, 56,7861

Received 27th November 2019,

Accepted 22nd January 2020

DOI: $10.1039 / \mathrm{c} 9 \mathrm{cc} 09249 \mathrm{~h}$

rsc.li/chemcomm

\section{Nickel(II)-methyl complexes adopting unusual seesaw geometries $\dagger$}

\author{
Ethan A. Hill, $\ddagger$ Norman Zhao, (D) $\ddagger$ Alexander S. Filatov (D) and John S. Anderson (D) *
}

\begin{abstract}
We report four-coordinate nickel(II)-methyl complexes of tris-carbene borate ligands which adopt rare seesaw geometries. Experimental and computational results suggest the structural distortion from threefold symmetry results from a combination of electronic stabilization of the singlet state, strong field donors, and constrained angles from the chelating ligand.
\end{abstract}

Nickel-alkyl complexes have been invoked as important intermediates in a host of chemical transformations including the production of acetic acid and cross-coupling reactions. ${ }^{1-6}$ In all reported examples of crystallographically characterized $\mathrm{Ni}(\mathrm{II})$-methyl complexes, a diamagnetic, square planar geometry is observed and many attempts to synthesize such species in other, four-coordinate geometries have proven unsuccessful frequently leading to reduction or other degradation. ${ }^{7-11}$ Despite this, catalytically active $\mathrm{Ni}(\mathrm{II})$-alkyl species may likely exhibit or transition through different coordination geometries. ${ }^{12-14}$ For example, other synthetic $\mathrm{Ni}(\mathrm{II})$ complexes can be found in tetrahedral geometries in addition to the more common square planar geometries. While a square planar geometry is favored due to electronic stabilization of the $\mathrm{d}^{8} \mathrm{Ni}(\mathrm{II})$ ion, a tetrahedral geometry may be favored with suitably bulky or chelating ligands. ${ }^{15}$

An alternative geometry for four-coordinate metal centers that has rarely been observed is a seesaw geometry. In fact, only a handful of examples of $\mathrm{Ni}$ (II) complexes in this geometry have been reported. ${ }^{13,16-18}$ In these cases, steric bulk is used to enforce the desired geometry. For example, the first of these reported by Bröring and co-workers utilized a tripyrranato ligand which positions methyl substituents within the square plane, forcing halide ligands above this plane. ${ }^{19-21}$ Other examples include those by Gossage and Baruah where homoleptic complexes of Ni(II) were synthesized with bulky propan-2-ylidene and

Department of Chemistry, The University of Chicago, 5735 S. Ellis Ave, Chicago, IL 60637, USA. E-mail: jsanderson@uchicago.edu

$\dagger$ Electronic supplementary information (ESI) available. CCDC 1968521-1968524. For ESI and crystallographic data in CIF or other electronic format see DOI: $10.1039 / \mathrm{c} 9 \mathrm{cc} 09249 \mathrm{~h}$

\# These authors contributed equally. oxazoline substituents that prevent planarization of the $\mathrm{Ni}(\mathrm{II})$ coordination environment. ${ }^{14,22}$ Another recent example of a seesaw geometry at a Ni(II) center involves a diisopropylpyrazole-substituted carbazole ligand to enforce steric crowding of the square plane. ${ }^{13}$ Finally, an example of a ligand-constrained geometry around a Ni(II) center can be seen in a complex bound to a triphosphacyclododecane ligand. ${ }^{23}$ This ligand binds in a facial manner with strongly donating trialkylphosphines which are proposed to enforce a low-spin state at nickel. Due to the constrained ligand environment, the coordination geometry distorts to accommodate the low-spin state but cannot fully isomerize to a square planar geometry. These examples demonstrate the lengths required to enforce this geometry at a $\mathrm{d}^{8} \mathrm{Ni}(\mathrm{II})$ center.

We have been interested in exploring the chemistry of late transition metals supported by chelating and strongly donating tris-N-heterocyclic carbene (NHC) borate scaffolds. ${ }^{24,25}$ These ligand scaffolds typically enforce a pseudo-tetrahedral geometry, but also favor low-spin states due to the strong donor properties of the carbene ligands. These factors suggest that $\mathrm{Ni}(\mathrm{II})$ complexes supported by this ligand would have geometric frustration between an electronically preferred square planar geometry and a chelate-enforced pseudo-tetrahedral geometry. We therefore rationalized that $\mathrm{Ni}(\mathrm{II})$ complexes of this ligand with suitably strong ligand fields might display unusual geometries and electronic structures. ${ }^{26-28}$ Here we report the isolation and characterization of two Ni(II)-methyl complexes supported by a strongly donating tris-carbene borate ligand which display unusual seesaw geometries.

The Ni(II)-chloride complexes were first synthesized by initial deprotonation of the proligand, $\left[\mathrm{PhB}(\mathrm{RImH})_{3}\right][\mathrm{OTf}]_{2}$ (where $\mathrm{R}={ }^{t} \mathrm{Bu}$ or $\mathrm{Ad}$ ), with in situ generated lithium diisopropylamide (LDA) followed by metalation with tetraethylammonium tetrachloronickelate $\left(\left[\mathrm{Et}_{4} \mathrm{~N}\right]_{2}\left[\mathrm{NiCl}_{4}\right]\right)$ to yield $\mathrm{PhB}\left({ }^{t} \mathrm{BuIm}\right)_{3} \mathrm{NiCl}$ and $\mathrm{PhB}(\mathrm{AdIm})_{3} \mathrm{NiCl}$ (1a and 1) ) in low but synthetically viable yields (see ESI $\dagger$ ). The title complexes, $\mathrm{PhB}\left({ }^{t} \mathrm{BuIm}\right)_{3} \mathrm{NiMe}$ and $\mathrm{PhB}(\operatorname{AdIm})_{3} \mathrm{NiMe}$ (2a and $\mathbf{2 b}$ ), were synthesized by treatment of the $\mathrm{Ni}(\mathrm{II})$-chloride complexes $\mathbf{1 a}$ and $\mathbf{1 b}$ with a solution of methyllithium following a similar reported procedure for related 

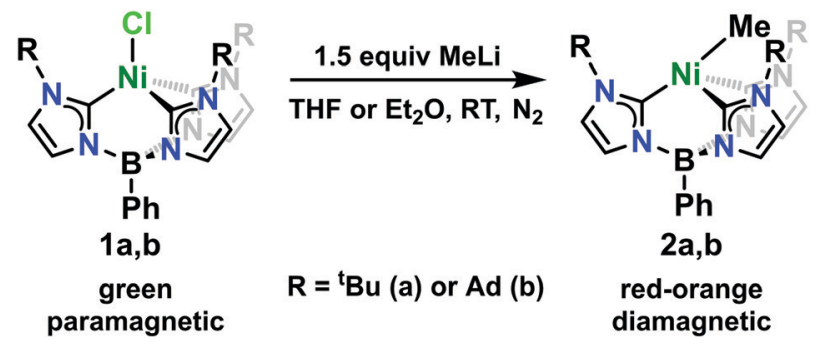

Scheme 1 Synthesis of complexes $\mathbf{2 a}$ and $\mathbf{2 b}$.

complexes of cobalt (Scheme 1). ${ }^{29}$ Complexes $2 \mathbf{a}$ and $\mathbf{2 b}$ can be isolated as red, microcrystalline solids in good yield. These complexes are thermally unstable at room temperature, but pure solids can be stored at $-35{ }^{\circ} \mathrm{C}$ for weeks to months without noticeable decomposition. Despite their intense, red-orange color in solution, complexes $\mathbf{2 a}$ and $\mathbf{2 b}$ display no distinct absorption features by UV-vis spectroscopy with only trailing absorbances from the UV region of the spectra into the visible region. Contrary to the parent Ni(II)-chloride complexes $\mathbf{1 a}$ and $\mathbf{1} \mathbf{b}$, both complexes $\mathbf{2 a}$ and $\mathbf{2 b}$ are diamagnetic. However, ${ }^{1} \mathrm{H}$ NMR spectra of both the $\mathrm{Cl}$ and Me complexes in $\mathrm{C}_{6} \mathrm{D}_{6}$ indicate $C_{3}$-symmetric geometries in solution at room temperature (see $\mathrm{ESI} \dagger$ ).

We then turned to single-crystal X-ray diffraction (SXRD) measurements to probe the structures of these Ni complexes in the solid-state. As expected from their threefold symmetric NMR spectra, complexes $\mathbf{1 a}$ and $\mathbf{1 b}$ are pseudo-tetrahedral in the solid-state and have a $C_{3}$-axis with a $\mathrm{B}-\mathrm{Ni}-\mathrm{Cl}$ angle of $177.92(6)^{\circ}$ and $178.8(2)^{\circ}$, respectively (see ESI $\dagger$ ). In contrast, while the room temperature ${ }^{1} \mathrm{H}$ NMR spectra of the methyl complexes $\mathbf{2 a}$ and $\mathbf{2 b}$ are consistent with a $C_{3}$-symmetric structure, an unusual seesaw coordination geometry at the Ni(II) centers is observed in their solid-state structures (Fig. 1). The metrical parameters of the Ni centers in complexes $\mathbf{2 a}$ and $\mathbf{2 b}$ are consistent with a seesaw geometry around the Ni(II) center composed of three carbon donors from the tris-carbene borate ligand and a fourth from the bound methyl group. The Ni-methyl carbon atom distances are nearly identical between the two complexes at 1.965(2) and 1.959(2) $\AA$, respectively. Additionally, there is no evidence of agostic interactions between the Me hydrogen atoms and the Ni(II) center. The two widest C-Ni-C angles which describe the seesaw geometry are $177.19(8)^{\circ}$ and $121.49(8)^{\circ}$ for $2 \mathrm{a}$ and $177.80(7)^{\circ}$ and $121.25(7)^{\circ}$ for $\mathbf{2 b}$. Using both of these angles, a geometry index parameter $\tau_{4}$ can be calculated to describe the coordination environment of the $\mathrm{Ni}(\mathrm{II})$ center between square planar $\left(\tau_{4}=0\right)$ or tetrahedral $\left(\tau_{4}=1\right) .{ }^{30,31}$ For both $2 \mathbf{a}$ and $2 \mathbf{b}$, the $\tau_{4}$ parameter is calculated to be 0.43 , indicating a nearly perfect mono-vacant, trigonal bipyramidal or seesaw geometry around the $\mathrm{Ni}$ (II) center. These are the first such cases to be crystallographically characterized. $\S$

With the disparity between solid-state and solution structural data, we sought to better understand the dynamics of complexes $\mathbf{2 a}$ and $\mathbf{2 b}$ in solution. Variable temperature ${ }^{1} \mathrm{H}$ NMR experiments were conducted in $\mathrm{d}_{8}$-toluene to determine an isomerization barrier, $\Delta \mathrm{G}^{\ddagger}$ (Fig. 2). ${ }^{32,33}$ In these complexes, the barrier being measured represents the energy to reorient

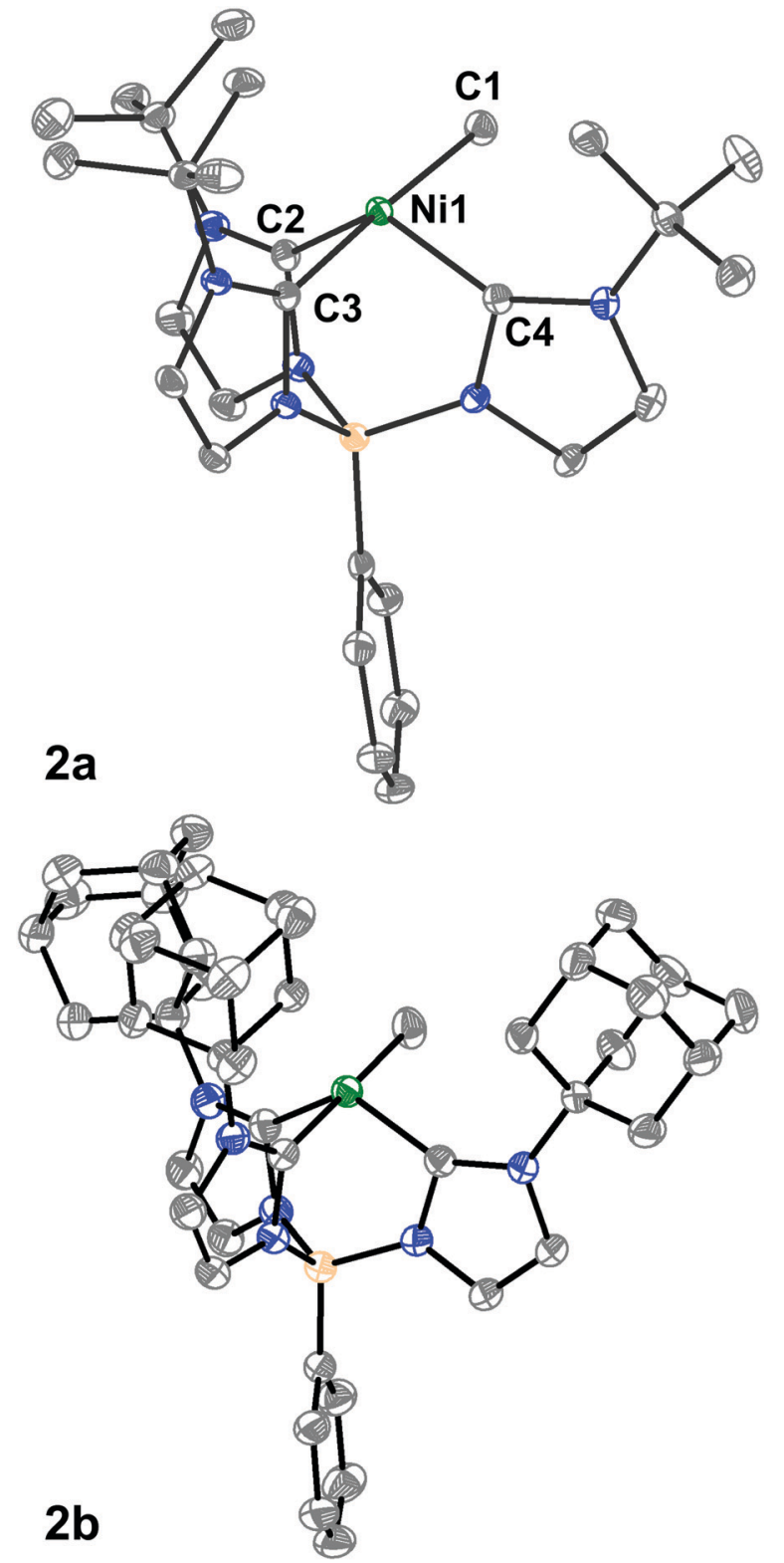

Fig. 1 SXRD structures of $\mathbf{2 a}$ and $\mathbf{2 b}$. Ellipsoids are shown at $50 \%$ and $\mathrm{H}$ atoms are omitted for clarity. Ni is shown in green, $\mathrm{N}$ in blue, B in tan, and $\mathrm{C}$ in gray. Atom labels for $2 \mathrm{~b}$ are the same as those depicted for $2 \mathrm{a}$. Selected bond lengths $(\AA)$ and angles $\left({ }^{\circ}\right)$ and geometric parameters for $\mathbf{2 a}$ : Ni1- $\mathrm{C} 1=$ 1.965(2), $\mathrm{Ni1}-\mathrm{C} 2=1.895(2), \mathrm{Ni1}-\mathrm{C} 3=1.922(2), \mathrm{Ni1}-\mathrm{C} 4=1.887(2)$, $\mathrm{C} 1-\mathrm{Ni1}-\mathrm{C} 3=177.19(8), \mathrm{C} 2-\mathrm{Ni1}-\mathrm{C} 4=121.49(8), \tau_{4}=0.43$. For $2 \mathbf{b}: \mathrm{Ni1}-\mathrm{C} 1=$ 1.959(2), $\mathrm{Ni} 1-\mathrm{C} 2=1.886(2), \mathrm{Ni} 1-\mathrm{C} 3=1.912(2), \mathrm{Ni1}-\mathrm{C} 4=1.884(2)$, $\mathrm{C} 1-\mathrm{Ni1}-\mathrm{C} 3=177.79(7), \mathrm{C} 2-\mathrm{Ni1}-\mathrm{C} 4=121.25(7), \tau_{4}=0.43$.

the methyl substituent from between one pair of NHC groups to between a different pair through either an effective lever mechanism or via $\mathrm{B}-\mathrm{Ni}-\mathrm{Me}$ linearization to a $C_{3}$-symmetric isomer (see below). ${ }^{34}$ For an NHC resonance of $2 \mathbf{a}$ (labeled with an asterisk in Fig. 2), a coalescence temperature of $215 \mathrm{~K}$ was determined and from the value of $\Delta \nu$, the peak-to-peak splitting in $\mathrm{Hz}$ of the fully resolved asymmetric structure, a barrier to isomerization was calculated to be $10.4 \pm 0.5 \mathrm{kcal} \mathrm{mol}^{-1}$. In the case of $\mathbf{2 b}$ with the larger adamantyl groups, we anticipated a 


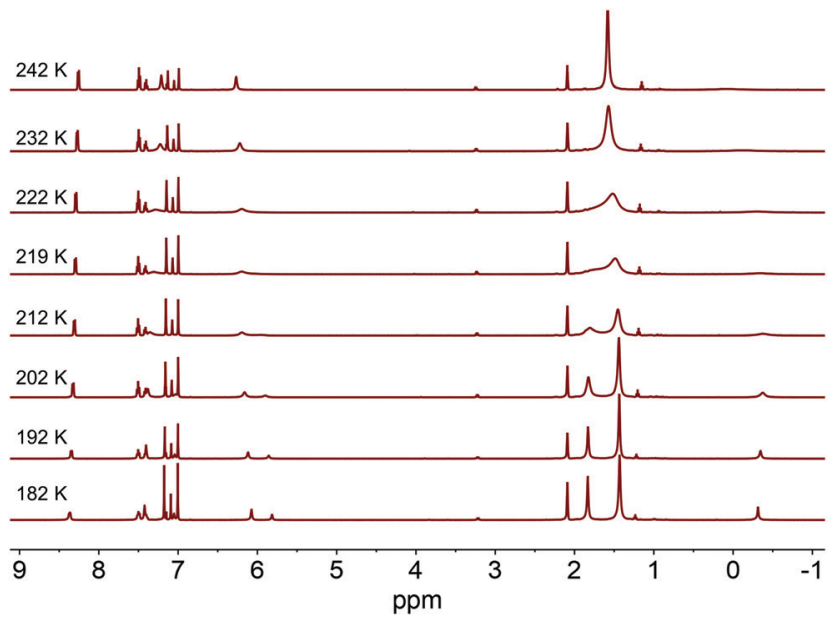

Fig. 2 Variable temperature ${ }^{1} \mathrm{H}$ NMR spectra for complex $\mathbf{2 a}$ in $\mathrm{d}_{8}$-toluene showing the splitting of the peaks of the imidazol-2-ylidene groups as temperature decreases. These data were used to determine coalescence temperature and calculate $\Delta G^{\ddagger}$ for the isomerization process. Asterisk marks resonance used for in-text values.

higher calculated barrier. However, the calculated value for the analogous imidazol-2-ylidene proton resonance is the same within an estimate of the error. From the coalescence temperature of $225 \mathrm{~K}$ and the measured $\Delta \nu$ values, a barrier of $10.9 \pm$ $0.5 \mathrm{kcal} \mathrm{mol}^{-1}$ was calculated (see ESI $\dagger$ ). This suggests that while the adamantyl groups are bulkier, extending further away from the Ni center, this does not appreciably alter the barrier to isomerization in this complex.

A point of interest upon studying complexes $\mathbf{2 a}$ and $\mathbf{2 b}$ was to rationalize the observed seesaw structures in the solid-state. A crude examination of the geometries a four-coordinate Ni(II) complex may adopt indicates three possible isomers as discussed above: tetrahedral, square planar, and seesaw. The parent chloride complexes $\mathbf{1 a}$ and $\mathbf{1 b}$ are characterized as high-spin $S=1$ complexes in a pseudo-tetrahedral geometry. Given the additional strong field methyl ligand in complexes $\mathbf{2 a}$ and $\mathbf{2 b}$, it is likely that diamagnetic, low-spin Ni(II) species would be most stable. ${ }^{35}$ While low-spin $\mathrm{Ni}$ (II) complexes are typically found in square planar geometries, the chelate ring of the tris(carbene)borate scaffold precludes the complex from distortion to a square planar geometry that would be otherwise electronically favored. Additionally, this stronger ligand field disfavors pseudo-tetrahedral geometries, as this would qualitatively result in occupation of a high energy, antibonding orbital (Fig. 3). As a result, the intermediate seesaw geometry is most stable, distorted away from pseudo-tetrahedral but restricted from planarization.

This qualitative description of the preference for seesaw geometries observed in complexes $\mathbf{2 a}$ and $\mathbf{2 b}$ is also supported by density functional theory (DFT) calculations. The geometry optimization of $\mathbf{2 a}$ in a singlet electronic configuration yields a structure that is in agreement with its solid-state structure. Geometry optimization of $\mathbf{2 a}$ in a triplet state shows instead a pseudo-tetrahedral geometry, similar to the geometry seen in 1a and 1b (see ESI $\dagger$ ). The DFT calculations predict the $S=0$ seesaw geometry to be $31 \mathrm{kcal} \mathrm{mol}^{-1}$ lower in energy than the

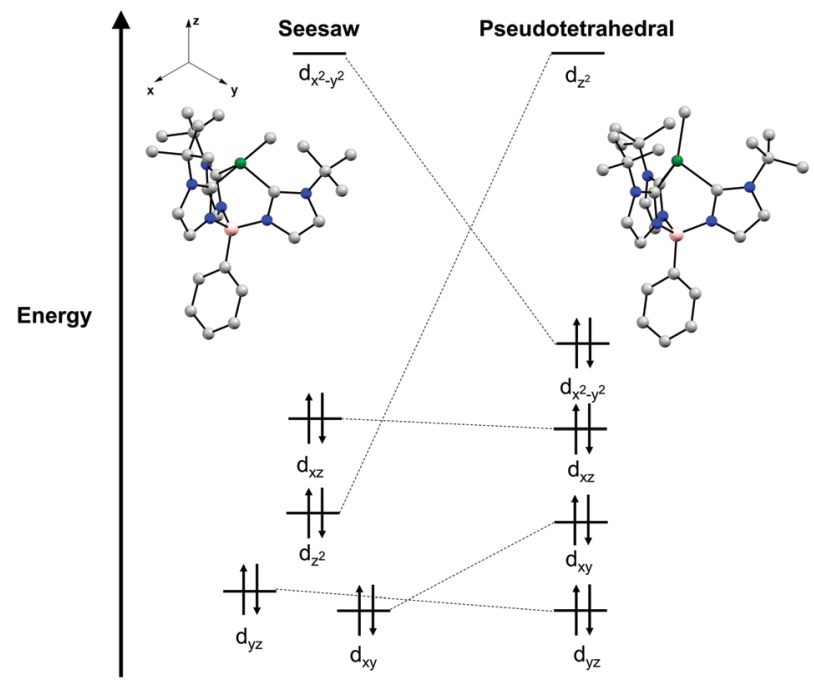

Fig. 3 Qualitative Walsh diagram depicting the relative energies of the d-orbitals for the seesaw and pseudo-tetrahedral geometries determined from DFT calculations of the geometry optimized singlet of $\mathbf{2 a}$. Basis set/ functional used: def2-TZVPP on Ni, N, and C bound to Ni, def2-SVP on all other atoms/O3LYP.

corresponding $S=0$ pseudo-tetrahedral isomer but only $11 \mathrm{kcal} \mathrm{mol}{ }^{-1}$ lower than the $S=1$ threefold symmetric species (see ESI $\dagger$ ). Qualitative comparison of the frontier molecular orbitals of singlet 2 a reveals that deviation from a pseudotetrahedral to seesaw geometry stabilizes the $\mathrm{d}_{z^{2}}$ orbital (Fig. 3). This results in a single, high-lying $\mathrm{d}_{x^{2}-y^{2}}$ orbital that remains unoccupied with a clustering of occupied orbitals at overall lower energy than those in the pseudo-tetrahedral geometry. This qualitative orbital depiction for the seesaw geometry is similar to the classical depiction of square planar d-orbital splitting, further suggesting such a geometry would be preferred in this system if not for the chelate ring constraint imposed by the tris-carbene borate ligand.

The DFT calculations above suggest that an isomerization mechanism involving a linear $S=1$ transition state is energetically reasonable, but we also wanted to probe the feasibility of a lever type mechanism for this isomerization computationally. In this mechanism the Me group migrates between NHC groups without linearizing to a pseudo-tetrahedral symmetry (see ESI $\dagger$ ). ${ }^{34}$ The $\mathrm{C}_{\mathrm{Me}}-\mathrm{Ni}-\mathrm{C}_{\text {carbene }}-\mathrm{N}_{\text {carbene }}$ dihedral angle was held fixed at $0^{\circ}$ and the Ni-Me group allowed to move between a pseudo-tetrahedral orientation and close proximity to the R-group of the NHC in order to find a low energy intermediate which would model the transition state for this isomerization (see ESI $\dagger$ ). In doing so, a conformer higher in energy by $20 \mathrm{kcal} \mathrm{mol}^{-1}$ relative to the seesaw geometry was found. This energy is high enough to rule out this pathway as operative given the $\sim 10 \mathrm{kcal} \mathrm{mol}^{-1}$ energy barrier calculated from the variable temperature ${ }^{1} \mathrm{H}$ NMR experiments. Given the computed relative energies of the $S=0\left(+31 \mathrm{kcal} \mathrm{mol}^{-1}\right)$ and $S=1\left(+11 \mathrm{kcal} \mathrm{mol}^{-1}\right)$ pseudo-tetrahedral isomers, it is likely that the isomerization proceeds through the $S=1 C_{3}$-symmetric isomer. This conclusion is also supported by the relatively broad $\mathrm{Ni}-\mathrm{CH}_{3}$ proton resonance at room temperature that shifts with 
temperature displaying Curie behavior (see ESI $\dagger$ ). This effect is likely the result of perturbation from a minor amount of the paramagnetic, pseudo-tetrahedral isomer at room temperature as has been previously observed. ${ }^{36}$ Interestingly, this observation suggests that paramagnetic Ni-methyl intermediates are accessible at room temperature in the current system, and that similarly high-spin Ni(II)-alkyl complexes may be reasonable intermediates in catalysis.

The Ni(II)-methyl complexes $\mathbf{2 a}$ and $\mathbf{2 b}$ are the first examples of such species to be found in a seesaw coordination geometry as revealed by their solid-state structures. Variable temperature ${ }^{1} \mathrm{H}$ NMR spectroscopy demonstrates that this distortion also occurs in the solution state and has an electronic origin as opposed to arising from any solid-state crystal packing. Using DFT calculations, the observed structures are rationalized by an overall lowering of filled orbitals by distortion from pseudotetrahedral to a seesaw geometry. This geometry is proposed to be favored over square planar geometry due to the chelate constraint of the tris(carbene)borate ligand. This work shows that Ni-alkyl species with chelating ligands can adopt this geometry and draws attention to the potential effects of this distortion and electronic structure on the reactivity of Ni intermediates in catalytic transformations.

This work was supported by an NSF CAREER award to JSA: Grant No. 1654144. NZ is supported by the National Science Foundation Graduate Research Fellowship under Grant No. DGE-1746045. NSF's ChemMatCARS Sector 15 is principally supported by the Divisions of Chemistry (CHE) and Materials Research (DMR), National Science Foundation, under grant number NSF/CHE-1834750. Use of the Advanced Photon Source, an Office of Science User Facility operated for the U.S. Department of Energy (DOE) Office of Science by Argonne National Laboratory, was supported by the U.S. DOE under Contract No. DE-AC02-06CH11357.

\section{Conflicts of interest}

There are no conflicts to declare.

\section{Notes and references}

$\S$ Determined by searching the Cambridge Crystallographic Database using Conquest by limiting a 4-coordinate Ni center with two pairs of ligand bond angles restricted between $150-180^{\circ}$ and $110-130^{\circ}$

1 P. A. Lindahl, J. Biol. Inorg. Chem., 2004, 9, 516-524.

2 X. Zhao, H.-Y. Tu, L. Guo, S. Zhu, F.-L. Qing and L. Chu, Nat. Commun., 2018, 9, 3488.

3 L. Guo and M. Rueping, Acc. Chem. Res., 2018, 51, 1185-1195.
4 J. Hu, Y. Zhao, J. Liu, Y. Zhang and Z. Shi, Angew. Chem., Int. Ed., 2016, 55, 8718-8722.

5 D. Nianios, S. Thierbach, L. Steimer, P. Lulchev, D. Klostermeier and S. Fetzner, BMC Biochem., 2015, 16, 10.

6 M. Can, F. A. Armstrong and S. W. Ragsdale, Chem. Rev., 2014, 114, 4149-4174.

7 E. Carmona, F. González, M. L. Poveda, J. L. Atwood and R. D. Rogers, J. Chem. Soc., Dalton Trans., 1980, 2108-2116.

8 P. Stoppioni, P. Dapporto and L. Sacconi, Inorg. Chem., 1978, 17, 718-725.

9 C. E. MacBeth, J. C. Thomas, T. A. Betley and J. C. Peters, Inorg. Chem., 2004, 43, 4645-4662.

10 P. J. Schebler, B. S. Mandimutsira, C. G. Riordan, L. M. Liable-Sands, C. D. Incarvito and A. L. Rheingold, J. Am. Chem. Soc., 2001, 123, 331-332.

11 N. Shirasawa, T. T. Nguyet, S. Hikichi, Y. Moro-oka and M. Akita, Organometallics, 2001, 20, 3582-3598.

12 S. A. Macgregor, Z. Lu, O. Eisenstein and R. H. Crabtree, Inorg. Chem., 1994, 33, 3616-3618.

13 J. Ghannam, T. Al Assil, T. C. Pankratz, R. L. Lord, M. Zeller and W.-T. Lee, Inorg. Chem., 2018, 57, 8307-8316.

14 A. Tarai and J. B. Baruah, Dalton Trans., 2018, 47, 4921-4930.

15 T. A. Albright, J. K. Burdett and M.-H. Whangbo, Orbital Interactions in Chemistry, John Wiley \& Sons, Inc., Hoboken, NJ, USA, 2nd edn, 2013.

16 C. A. Grapperhaus, J. A. Bellefeuille, J. H. Reibenspies and M. Y. Darensbourg, Inorg. Chem., 1999, 38, 3698-3703.

17 R. Graziani, M. Vidali, U. Casellato and P. A. Vigato, Transition Met. Chem., 1978, 3, 99-103.

18 J. Li, D. Tian, H. Song, C. Wang, X. Zhu, C. Cui and J. P. Cheng, Organometallics, 2008, 27, 1605-1611.

19 M. Bröring, S. Prikhodovski and C. D. Brandt, J. Chem. Soc., Dalton Trans., 2002, 4213-4218.

20 M. Bröring, S. Prikhodovski and C. D. Brandt, Inorg. Chim. Acta, 2004, 357, 1733-1738.

21 M. Bröring, S. Prikhodovski, C. D. Brandt and E. C. Tejero, Chem. - Eur. J., 2007, 13, 396-406.

22 J. A. Adjei, A. J. Lough and R. A. Gossage, RSC Adv., 2019, 9, 3956-3964.

23 P. G. Edwards, F. Ingold and M. B. Hursthouse, Chem. Commun., 1998, 545-546.

24 M. K. Goetz, E. A. Hill, A. S. Filatov and J. S. Anderson, J. Am. Chem. Soc., 2018, 140, 13176-13180.

25 M. K. Goetz and J. S. Anderson, J. Am. Chem. Soc., 2019, 141, 4051-4062.

26 R. A. Juarez, W.-T. Lee, J. M. Smith and H. Wang, Dalton Trans., 2014, 43, 14689-14695.

27 I. Nieto, R. P. Bontchev and J. M. Smith, Eur. J. Inorg. Chem., 2008, 2476-2480.

28 S. B. Muñoz, W. K. Foster, H.-J. Lin, C. G. Margarit, D. A. Dickie and J. M. Smith, Inorg. Chem., 2012, 51, 12660-12668.

29 R. E. Cowley, R. P. Bontchev, E. N. Duesler and J. M. Smith, Inorg. Chem., 2006, 45, 9771-9779.

30 L. Yang, D. R. Powell and R. P. Houser, Dalton Trans., 2007, 955-964.

31 D. Rosiak, A. Okuniewski and J. Chojnacki, Polyhedron, 2018, 146, 35-41.

32 H. Shanan-Atidi and K. H. Bar-Eli, J. Phys. Chem., 1970, 74, 961-963.

33 J. Sandström, Dynamic NMR Spectroscopy, Academic Press, London, 1982.

34 M. Mauksch and P. v. R. Schleyer, Inorg. Chem., 2001, 40, 1756-1769.

35 J. Cirera, E. Ruiz and S. Alvarez, Inorg. Chem., 2008, 47, 2871-2889.

36 B. Le Guennic, T. Floyd, B. R. Galan, J. Autschbach and J. B. Keister, Inorg. Chem., 2009, 48, 5504-5511. 